\title{
Evidence for Anomalous Thermal Expansion at a Crystal Surface
}

\author{
Joost W. M. Frenken, F. Huussen, and J. F. van der Veen \\ FOM-Institute for Atomic and Molecular Physics, 1098 SJ Amsterdam, The Netherlands
}

(Received 20 June 1986)

\begin{abstract}
Ion shadowing and blocking measurements indicate that the thermal expansion coefficient of a $\mathrm{Pb}$ crystal is substantially enhanced at its (110) surface. This is evidenced by a decrease in surface contraction with temperature. The first atomic layer is shifted with respect to its truncated-bulk location by $-(15.4 \pm 2.5) \%$ of a bulk spacing at $29 \mathrm{~K}$, but by only $-(3 \pm 5) \%$ at $485 \mathrm{~K}$.
\end{abstract}

PACS numbers: $65.70 .+\mathrm{y}, 61.80 . \mathrm{Mk}, 68.35 . \mathrm{Bs}$

Nowadays, theoretical predictions and experimental determinations of multilayer relaxation at low-index metal surfaces are at a level of quantitative agreement. ${ }^{1-3}$ In general, the first few interlayer distances are relaxed, in a damped oscillatory manner. ${ }^{1,2}$ So far, most experiments have been conducted at temperatures sufficiently low that the neglect of thermal vibrations in the theoretical treatment of relaxation appears justified. As the temperature rises, however, the amplitude of lattice vibrations increases and the anharmonic terms in the interatomic potential become more important. Minimization of the anharmonic free energy of the crystal with respect to the interlayer distances then leads to the prediction of thermal expansion. As the thermal vibration amplitude of atoms in the surface is much larger than in the bulk, the thermal expansion coefficient is expected to be enhanced at the surface, typically by a factor of 2 or more. ${ }^{4,5}$

While the phenomenon of thermal expansion as an important material property has been studied extensively in the bulk, at the surface it has essentially remained undetected for lack of a direct probe of near-surface interlayer spacings. Analyses of low-energy electron diffraction experiments, in which a value for the surface expansion coefficient was deduced from minor shifts in Bragg peaks, ${ }^{6-8}$ proved largely unfounded, because multiple-scattering effects were neglected. So far, a single, indirect indication of enhanced thermal expansion at a crystal surface has been obtained from spin-polarized low-energy electron diffraction experiments, ${ }^{9}$ where a temperature-dependent energy shift of the spinpolarization pattern was found to be slightly larger than the shift which was calculated for bulk expansion.

In this Letter the first direct evidence is presented for anomalous thermal expansion at a crystal surface. Ion backscattering measurements on the $\mathrm{Pb}(110)$ surface reveal a strong lattice contraction at low temperature: At $29 \mathrm{~K}$ the shift $\Delta d_{1 b} / d$ of the first layer with respect to its truncated-bulk position equals $-(15.4 \pm 2.5) \%$ of the bulk spacing. Upon heating, the contraction decreases (i.e., the surface expands) nonlinearly to $\Delta d_{1 b} / d=-(3$ $\pm 5) \%$ at $485 \mathrm{~K}$, at which temperature the surface becomes partially disordered. ${ }^{10}$ The data are suggestive of a correlation between the vanishing contraction and the onset of surface premelting effects, as predicted by Jayanthi, Tosatti, and Pietronero. ${ }^{5}$

$\mathrm{Pb}$ specimens were spark cut from a single-crystal $\mathrm{Pb}$ bar. Chemical polishing and sputter cleaning in ultrahigh vacuum at elevated temperature produced a well-ordered surface, free from impurities. The crystal container was connected, via a $\mathrm{Cu}$ braid, to a He-flow cryostat, ${ }^{11}$ with which crystals were cooled to $29 \mathrm{~K}$ within $9 \mathrm{~min}$. Crystals were heated by electron bombardment or radiative heating of the back of their container.

The experimental technique of ion backscattering in conjunction with shadowing and blocking has been described previously. ${ }^{12}$ Figure 1 illustrates the principle of the relaxation measurement. A parallel beam of 97.6$\mathrm{keV}$ protons is aligned with the [101] axis of the $\mathrm{Pb}$ crystal. In this geometry, the large surface contraction makes the $\mathrm{Pb}$ atoms in the first and the second layers fully visible to the impinging protons. Atoms in deeper layers are shadowed and hence contribute much less strongly to the backscattering yield. Protons backscattered from atoms in the second layer are blocked on their way

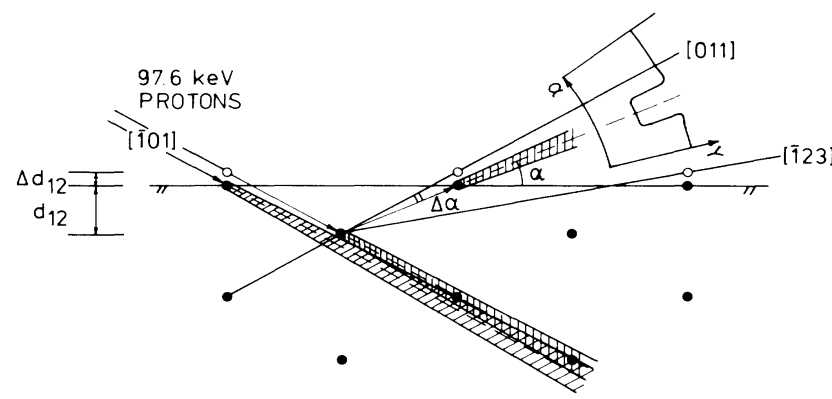

FIG. 1. Side view of the (111) scattering plane perpendicular to the $\mathrm{Pb}(110)$ surface. Bulk lattice sites are indicated by open circles. Solid circles denote atoms in a contracted top layer. The principle of the measurement is schematically shown. The vertical lines denote the directions of the [011] and [123] bulk crystal axes at $30^{\circ}$ and $10.9^{\circ}$, respectively (at each temperature the direction of the [011] bulk axis was measured to within $\pm 0.03^{\circ}$, and used as a reference angle). 
out by the top-layer atoms in directions near the [011] and [123] crystal axes. A toroidal electrostatic energy analyzer records simultaneously the surface backscattering yield $Y$ (Fig. 1) over a $20^{\circ}$ range of scattering angles around a given blocking direction. The yields are calibrated to within $\pm 3 \%$ to give the number of visible $\mathrm{Pb}$ monolayers as a function of exit angle $\alpha$, i.e., the "surface blocking pattern." Surface relaxation gives rise to an angular shift $\Delta \alpha$ of the surface blocking pattern with respect to the bulk crystal axis.

If the atoms in the third and deeper layers are perfectly shadowed, as is the case for very small thermal vibration amplitudes, the percentage change in the first interlayer spacing $\Delta d_{12} / d$ follows immediately from the geometric relation $\Delta d_{12} / d=\tan \left(\alpha_{b}+\Delta \alpha\right) / \tan \left(\alpha_{b}\right)-1$, where $\alpha_{b}$ is the angle of the corresponding bulk axis with respect to the surface plane. The bottom curve in Fig. 2 is the surface blocking pattern measured in the geometry of Fig. 1 at a temperature of $29 \mathrm{~K}$. At this temperature, the bulk vibration amplitude $\sigma_{b}$ equals $0.057 \AA,{ }^{13,14}$ which is close to the zero-point value of $0.043 \AA$. At exit angles $\alpha$ between $12^{\circ}$ and $20^{\circ}$, and at $\alpha$ above $30^{\circ}$, the first two atomic layers are fully visible to the ion beam and detector. Around $25.1^{\circ}$ and $8.8^{\circ}$ the backscattering yield from the second layer is almost entirely blocked. From the angular shifts of $\Delta \alpha=-4.9^{\circ}$ and $-2.1^{\circ}$ of these surface blocking directions with respect to the corresponding [011] and [1 23 ] bulk axes, we calculate a surface contraction of $\Delta d_{12} / d \approx-19 \%$. A small correction is made to account for the fact that the average inflection point of protons backscattering from secondlayer atoms lies somewhat closer to the surface than the second-layer atoms themselves. ${ }^{15,16}$ This results in $\Delta d_{12} / d=-17.2 \%$. The measurements are also sensitive to $\Delta d_{23} / d$ (as a result of a 0.06 visible-layer backscattering contribution from the third layer), and to the ratio $S$

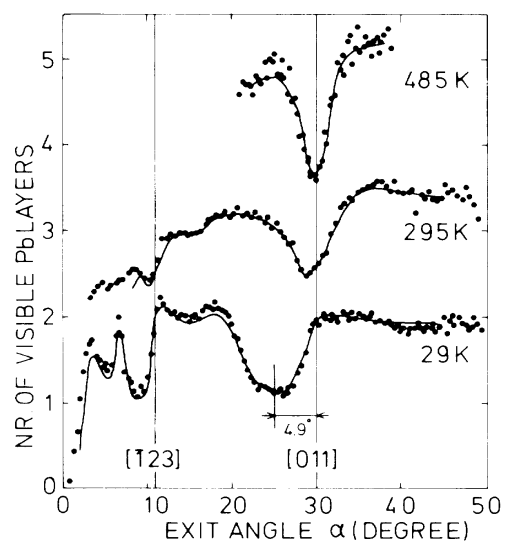

FIG. 2. Surface blocking patterns (circles), measured with 97.6-keV protons, in the geometry of Fig. 1, at temperatures of 29, 295, and $485 \mathrm{~K}$. Curves are the best-fit results of Monte Carlo computer simulations (see text). between surface and bulk vibration amplitudes. To extract this extra information from the data, Monte Carlo computer simulations of the backscattering experiment ${ }^{15}$ were performed for a range of assumed relaxation parameters and vibration amplitudes. ${ }^{13}$ The best fit to the experimental data was obtained for $\Delta d_{12} / d=-(17.2$ $\pm 0.5) \%, \Delta d_{23} / d=+(8.0 \pm 2.0) \%$, and $S=1.5 \pm 0.1$. The fit is shown in Fig. 2 by a solid curve. It closely matches the experimental data over the entire range of exit angles. Recently, a total-energy minimization calculation, based on pseudopotential theory of simple metals, has been performed to predict surface relaxation parameters for $\mathrm{Pb}(110)$ at $T=0 .{ }^{16}$ The theoretical values of $\Delta d_{12} / d=-15.9 \%, \quad \Delta d_{23} / d=+7.9 \%, \quad \Delta d_{34} / d=-6.8 \%$, and $\Delta d_{45}=+0.7 \%$ (deeper-layer relaxations are negligible) are in excellent agreement with experiment. Complementing our experimental values for $\Delta d_{12} / d$ and $\Delta d_{23} / d$ with the theoretical values for $\Delta d_{34} / d$ and $\Delta d_{45} / d$, a net relaxation of $\Delta d_{1 b} / d=\sum \Delta d_{i, i+1} / d=-(15.4$ $\pm 2.5) \%$ is obtained. The $2.5 \%$ error margin is the sum of the experimental uncertainties in $\Delta d_{12} / d$ and $\Delta d_{23} / d$.

At high temperatures, the large vibration amplitudes $\left(\sigma_{b}=0.18\right.$ and $0.24 \AA$ at 295 and $485 \mathrm{~K}$, respectively ${ }^{14}$ ) cause the backscattering contributions from third- and deeper-layer atoms to increase substantially. These contributions are blocked in directions which depend not only on $\Delta d_{12} / d$ but also on $\Delta d_{23} / d, \Delta d_{34} / d$, etc. The resulting surface blocking pattern is in general asymmetric and the angular shift $\Delta \alpha$ of its minimum takes an intermediate value which corresponds to a linear combination of $\Delta d_{12} / d, \Delta d_{23} / d$, etc. A geometric analysis in terms of the individual relaxations is then no longer tractable. At a temperature of $295 \mathrm{~K}$ (Fig. 2, middle curve) the asymmetry of the minimum is strong enough that individual relaxations can be determined by a comparison of the measured blocking patterns with Monte Carlo simulations. The analysis of the surface blocking measurement at $295 \mathrm{~K}$ has been presented elsewhere. ${ }^{16}$ The blocking patterns were found to be sensitive to $\Delta d_{12} / d$ and to a linear combination of $\Delta d_{23} / d$ and $\Delta d_{34} / d$. The best fit (Fig. 2) was obtained for $\Delta d_{12} / d=-(15.8 \pm 2.5) \%$, $\Delta d_{23} / d+0.75 \Delta d_{34} / d=+(0.5 \pm 2.5) \%, \quad$ and $S=1.5$ \pm 0.1 . Within the error margins, the relaxation parameters correspond well with both the theory and the experimental relaxation at $29 \mathrm{~K}$. Note that the large angular shift of the surface blocking minimum relative to the one of $29 \mathrm{~K}$ does not reflect a temperature-dependent relaxation effect, but instead an increased sampling over deeper layers. Combining the $295-\mathrm{K}$ value for $\Delta d_{12} / d$ with the $29-\mathrm{K}$ value for $\Delta d_{23} / d$ and the theoretical values for $\Delta d_{34} / d$ and $\Delta d_{45} / d$, we find $\Delta d_{1 b} / d=-(14.0 \pm 4.5) \%$ at this temperature. The error bar again reflects the sum of the uncertainties in the experimental relaxations.

Finally, at $485 \mathrm{~K}$ more than ten layers contribute to backscattering, resulting in a yield of $\sim 5$ visible layers outside the blocking direction (Fig. 2, top curve). The nearly symmetric blocking pattern is much less sensitive 
to the individual relaxations than the patterns at lower temperature. Its blocking is essentially determined by the sum of the multilayer relaxations, i.e., the net relaxation $\Delta d_{1 b} / d$. To facilitate a comparison with Monte Carlo simulations, the same data are plotted in Fig. 3 on an expanded angular scale. The dashed curve in Fig. 3 shows the surface blocking pattern which was simulated with the assumption of the low-temperature structure $\Delta d_{12} / d=-17.2 \%, \quad \Delta d_{23} / d=+8.0 \%, \Delta d_{34} / d=-6.8 \%$, and $\Delta d_{45} / d=+0.7 \%$, i.e., $\Delta d_{1 b} / d=-15.4 \%$. The ratio between surface and bulk vibration amplitudes was again taken as $S=1.5$. The measured surface blocking minimum occurs at a $0.2^{\circ}$ higher exit angle than the simulated one (dashed curve). This small difference is significant in view of the fact that the experimentally determined angles are calibrated to within $0.03^{\circ}$. Also for other sets of relaxation values chosen within the error margins of the experiments at 29 and $295 \mathrm{~K}$, the simulated minima do not fit the angular position of the measured one. The simulations produce the minimum at the correct angle only if the net relaxation $\Delta d_{1 b} / d$ is taken to be $-(3 \pm 5) \%$. The best fit for $\Delta d_{1 b} / d=-3 \%$ is shown in Figs. 2 and 3. The error margin reflects the maximum variations in $\Delta d_{1 b} / d$ as a result of experimental uncertainties in the calibration, the value of $S$, and the bulk vibration amplitude $\sigma_{b}$. It is concluded that the surface undergoes an enhanced thermal expansion of $\delta\left(\Delta d_{1 b} / d\right)$ $=(-3)-(-15)=12 \%$ over the temperature range between $29 \mathrm{~K}$ and $485 \mathrm{~K}$. Since the measurement at $485 \mathrm{~K}$ is insensitive to the individual relaxation values $\Delta d_{i, i+i} / d$, we cannot determine whether this expansion is confined to the first interlayer distance, or distributed over several interlayer distances. Theoretically, the excess thermal expansion is expected to be largely concentrated in the first. ${ }^{4}$ Note that our method of measuring temperaturedependent relaxations is not sensitive to bulk thermal expansion, but exclusively to the difference in expansion between surface and bulk.

In Fig. 4 the relaxation $\Delta d_{1 b} / d$ is plotted as a function of temperature. Besides the values obtained from the

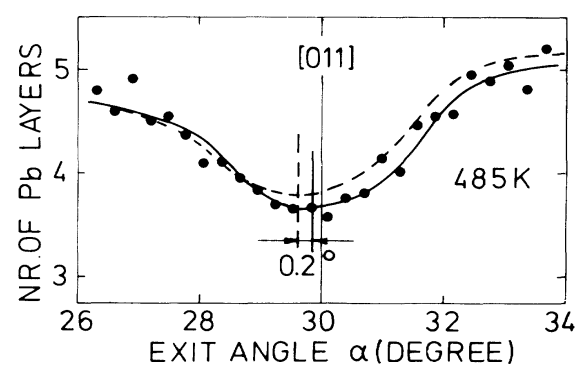

FIG. 3. Surface blocking pattern measured at $485 \mathrm{~K}$ (circles), plotted on an extended angle scale. The solid curve is the best fit for $\Delta d_{1 b} / d=-3 \%$, discussed in the text. The dashed curve is the result of a simulation for the low-temperature structure with $\Delta d_{1 b} / d=-15.4 \%$. blocking patterns in Fig. 2, three other values are shown for intermediate temperatures. Although the error bars in Fig. 4 partly overlap, the surface relaxation definitively shows a trend towards a reduced contraction at high temperatures. Blocking patterns measured at temperatures between $485 \mathrm{~K}$ and the melting point $(600.7 \mathrm{~K})$ have not been examined for $\Delta d_{1 b} / d$, since at these temperatures the surface is no longer well ordered, as a result of surface premelting. ${ }^{10}$ At $485 \mathrm{~K}$ the surface disorder is evident as a small uniform increase in surface backscattering yield by $\sim 1 / 10$ monolayer (Ref. 10 and Fig. 4). These few disorderly positioned atoms do not influence the blocking effect which originates from the ordered part of the surface region.

In the temperature range considered, bulk $\mathrm{Pb}$ has an almost constant thermal expansion coefficient of $28 \times 10^{-6} \mathrm{~K}^{-1},{ }^{17}$ and so the bulk expands by no more than $1.3 \%$ between $29 \mathrm{~K}$ and $485 \mathrm{~K}$. If the surface expansion coefficient is also constant with temperature, the observed changes in relaxation correspond to a surface expansion coefficient which is 3.5 to 12 times higher. The data in Fig. 4 suggest, however, that the surface expansion coefficient is not constant, but increases nonlinearly at high temperature. A similar behavior was predicted recently by Jayanthi, Tosatti, and Pietronero in a theoretical study of $\mathrm{Cu}$ surfaces. ${ }^{5}$ It was argued in this study that a rapid increase in surface expansion is a consequence of strong surface anharmonicity, leading eventually to an instability at the surface and premelting

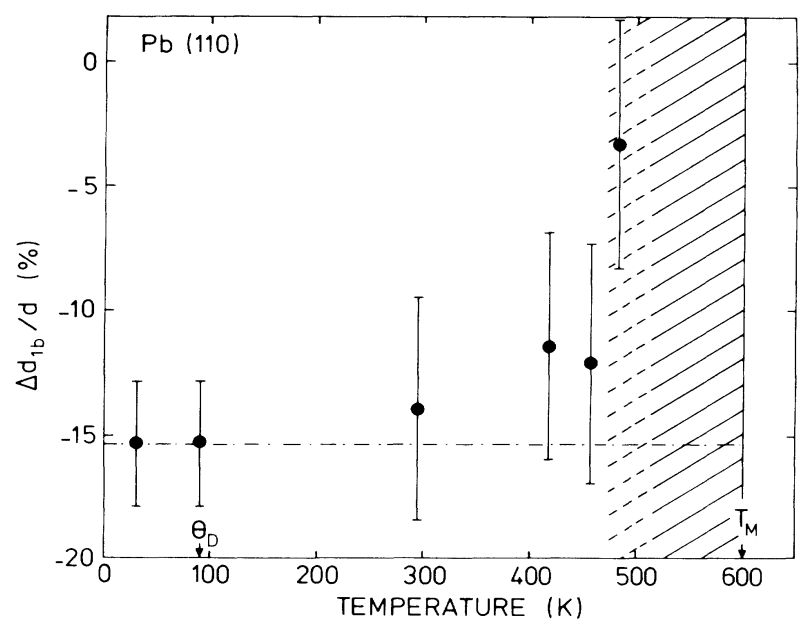

FIG. 4. The temperature-dependent relaxation $\Delta d_{1 b} / d$ of the first layer with respect to its bulk-truncated position. Arrows indicate the bulk Debye temperature $\theta_{\mathrm{D}}$ and the melting point $T_{m}$. A surface thermal expansion equal to the bulk expansion would result in the dash-dotted line. The surface premelting temperature regime (Ref. 10) is hatched. The dashed part of the hatched region marks temperatures where the surface region contains a small number of disorderly positioned atoms, increasing from the equivalent of 0.1 to 0.5 monolayer. 
of the first layer. Interestingly, above $\sim 485 \mathrm{~K}$, at which temperature the contraction of $\mathrm{Pb}(110)$ is seen to have almost vanished, the $\mathrm{Pb}(110)$ surface is also known to become gradually disordered and to exhibit premelting ${ }^{10}$ (indicated as hatched area in Fig. 4). The present data suggest that there indeed exists a correlation between the loss of surface contraction and the onset of disordering (premelting). This intriguing issue needs to be further explored.

A. J. Riemersma and P. H. M. van Berge Henegouwen of the University of Amsterdam are gratefully recognized for the careful preparation of our $\mathrm{Pb}$ specimens. This work is sponsored by Fundamenteel Onderzoek der Materie with financial support from the Nederlandse Organisatie voor Zuiver Wetenschappelijk Onderzoek.

${ }^{1}$ R. N. Barnett, U. Landman, and C. L. Cleveland, Phys. Rev. B 28, 1685 (1983), and references therein.

${ }^{2}$ K. M. Ho and K. P. Bohnen, Phys. Rev. B 32, 3446 (1985).

${ }^{3}$ J. W. M. Frenken, J. F. van der Veen, and G. Allan, Phys. Rev. Lett. 51, 1876 (1983).

${ }^{4}$ See, e.g., S. K. S. Ma, F. W. de Wette, and G. P. Alldredge, Surf. Sci. 78, 598 (1978), and references therein.

${ }^{5}$ C. S. Jayanthi, E. Tosatti, and L. Pietronero, Phys. Rev. B 31, 3456 (1985).

6P. E. Viljoen, B. J. Wessels, G. L. Benning, and J. P. Roux, J. Vac. Sci. Technol. 20, 204 (1982).

${ }^{7}$ E. N. Lubnin and Yu. Ya. Tomashpol'skii, Fiz. Tverd. Tela
(Leningrad) 23, 3697 (1981) [Sov. Phys. Solid State 23, 2157 (1981)].

${ }^{8}$ J. M. Wilson and T. J. Bastow, Surf. Sci. 26, 461 (1971).

9 J. Kirschner and R. Feder, Surf. Sci. 104, 448 (1981).

${ }^{10} \mathrm{~J}$. W. M. Frenken and J. F. van der Veen, Phys. Rev. Lett. 54, 134 (1985); J. W. M. Frenken, P. M. J. Marée, and J. F. van der Veen, Phys. Rev. B 34, 7506 (1986).

${ }^{11}$ F. Huussen, J. W. M. Frenken, and J. F. van der Veen, Vacuum 36, 259 (1986).

${ }^{12}$ J. F. van der Veen, Surf. Sci. Rep. 5, 199 (1985), and reference therein.

${ }^{13}$ In the Monte Carlo simulations the distributions of thermal displacements are approximated by Gaussians. Anharmonicity results in non-Gaussian thermal displacement distributions. However, ion blocking is mainly sensitive to the time-averaged position of an atom $\langle x\rangle$, and its root mean square onedimensional displacement $\sigma=\left[\left\langle(x-\langle x\rangle)^{2}\right\rangle / 3\right]^{1 / 2}$ from $\langle x\rangle$. We can therefore safely mimic the position distribution by a Gaussian, centered around $\langle x\rangle$, and having a second moment equal to $\sigma$ (quasiharmonic approximation). In the text $\sigma$ is referred to as the vibration amplitude.

${ }^{14}$ E. V. Zarochentsev, S. P. Kravchuk, and T. M. Tarusina, Fiz. Tverd. Tela (Leningrad) 18, 413 (1976) [Sov. Phys. Solid State 18, 239 (1976)], and references therein.

15 J. W. M. Frenken, R. M. Tromp, and J. F. van der Veen, Nucl. Instrum. Methods Phys. Res. Sect. B 17, 334 (1986); R. M. Tromp and J. F. van der Veen, Surf. Sci. 133, 159 (1983).

${ }^{16}$ J. W. M. Frenken, J. F. van der Veen, R. N. Barnett, U. Landman, and C. L. Cleveland, Surf. Sci. 172, 319 (1986).

${ }^{17}$ G. Borelius, in Solid State Physics, Advances in Research and Applications, edited by F. Seitz and D. Turnbull (Academic, New York, 1958). 